\title{
Comparative analysis of three types of minimally invasive decompressive surgery for lumbar central stenosis: biportal endoscopy, uniportal endoscopy, and microsurgery
}

\author{
*Dong Hwa Heo, MD, PhD, Dong Chan Lee, MD, and Choon Keun Park, MD, PhD \\ Department of Neurosurgery, Spine Center, The Leon Wiltse Memorial Hospital, Suwon, South Korea
}

\begin{abstract}
OBJECTIVE Recently, minimally invasive unilateral laminotomy with bilateral decompression (ULBD) has been performed for lumbar stenosis using endoscopic approaches. The object of this retrospective study was to compare the clinical and radiological outcomes of three types of minimally invasive decompressive surgery: microsurgery, percutaneous uniportal endoscopic surgery, and percutaneous biportal endoscopic surgery.

METHODS In the period from March 2016 to December 2017, minimally invasive ULBD was performed using microscopy, a uniportal endoscopic approach, or a biportal endoscopic approach to treat lumbar canal stenosis. Patients were classified into three groups based on the surgery they had undergone. The angle of medial facetectomy area and postoperative dural expansion were measured using MR images. The visual analog scale (VAS) score for leg and back pain, Oswestry Disability Index (ODI), operation time, and complications were assessed. Clinical and radiological parameters were compared among the three groups.
\end{abstract}

RESULTS There were 33 patients in the microscopy group, 37 in the biportal endoscopy group, and 27 in the uniportal endoscopy group. Preoperatively stenotic dural areas were significantly expanded in each of the three groups after surgery $(p<0.05)$. Mean dural expansion in the uniportal endoscopy group was significantly lower than that in the microscopy or biportal endoscopy group $(p<0.05)$. The mean angle of the facetectomy in the biportal endoscopic group was significantly lower than that in the microscopic group or uniportal endoscopic group $(p<0.05)$. On the 1 st day after surgery, the VAS score for back pain was significantly higher in the microscopic group than in the uniportal or biportal endoscopic group $(p<0.05)$. However, there were no significant differences in the VAS score for back pain, VAS score for leg pain, or ODI at the final follow-up among the three groups $(p>0.05)$.

CONCLUSIONS Although radiological results were different among the three groups of patients, postoperative clinical outcomes were significantly improved after each type of surgery. The percutaneous biportal or uniportal endoscopic approach offers the advantage of reduced immediate postoperative pain. A percutaneous uniportal or biportal endoscopic lumbar approach may be effective for the treatment of lumbar central stenosis and an alternative to conventional microsurgical decompression.

https://thejns.org/doi/abs/10.3171/2019.2.FOCUS197

KEYWORDS surgical endoscopy; surgical decompression; minimally invasive surgery; stenosis; magnetic resonance imaging

$\mathrm{L}$

UMBAR spinal stenosis is one of the lumbar degenerative diseases presenting with radicular leg pain, tion. ${ }^{15}$ The first treatment for symptomatic lumbar stenosis is conservative management including medication, physical therapy, and nerve-block procedures. ${ }^{15}$ Surgical treatments are considered for patients with severe radicular pain and walking disability refractory to conservative management. ${ }^{8}$ Decompressive surgery may be effective in improving radicular pain and the quality of life of the patient. ${ }^{4}$ Recently, minimally invasive spine surgery via unilateral laminotomy with bilateral decompression (ULBD) has been per-

ABBREVIATIONS ODI = Oswestry Disability Index; ULBD = unilateral laminotomy with bilateral decompression; VAS = visual analog scale.

SUBMITTED January 2, 2019. ACCEPTED February 26, 2019.

INCLUDE WHEN CITING DOI: 10.3171/2019.2.FOCUS197.

* D.H.H. and D.C.L. contributed equally to this work and share first authorship. 
formed to minimize trauma to the posterior musculo-ligamentous structures and prevent postoperative segmental instability, which can occur in traditional decompressive surgeries such as subtotal laminectomy., ${ }^{3,13,14}$ Endoscopic spinal surgeries are minimally invasive as well. Their indications have been extended to include lumbar stenosis. Various endoscopic techniques have been tried to treat lumbar central stenosis and foraminal stenosis.7,9,1

It has been reported that endoscopic decompressive surgeries, including percutaneous uniportal and biportal endoscopic approaches, have good clinical results. ${ }^{1,9,12}$ However, few studies have reported the radiological outcomes of those approaches compared to those of conventional decompressive surgeries. ${ }^{6,10}$ In addition, there are few studies on whether endoscopic decompression for lumbar stenosis can achieve complete neural decompression like the open decompressive surgeries. ${ }^{6}$

Therefore, the object of the present study was to compare the clinical and radiological outcomes of three types of minimally invasive decompressive surgery: microsurgery, percutaneous uniportal endoscopic surgery, and percutaneous biportal endoscopic surgery. Neural decompression such as dural expansion after surgery was assessed by measuring the decompressed neural area on MRI.

\section{Methods}

This study was approved by the institutional review board of our hospital. It was a retrospective analysis of prospectively collected data on patients treated in the period from March 2016 to December 2017. All patients reported claudication or pain radiating to the lower extremities that had been refractory to conservative treatments including physical therapy, medication, and epidural injection procedures for at least 12 weeks. ${ }^{6}$ Inclusion criteria were as follows: 1) lumbar central and lateral recess stenosis; 2) single-level stenosis at L4-5; and 3) follow-up for more than 1 year. Patients who had foraminal stenosis, infectious disease from a thoracolumbar lesion, concomitant segmental instability, spondylolisthesis, revision surgeries at the same spinal level, concomitant traumatic conditions, or musculoskeletal disorders were excluded. ${ }^{6}$

Five experienced spine surgeons performed all surgical procedures. All five surgeons have at least 10 years of microscopic surgery experience and at least 5 years of endoscopic surgery experience. The operative procedure was determined according to the preference and experience of the operating surgeon. We provided information about the surgeries to each patient beforehand.

We performed three kinds of minimally invasive ULBD: microscopic ULBD using a spinal microscopic system; biportal endoscopic ULBD using percutaneous biportal endoscopic systems (Fig. 1 upper); and uniportal endoscopic ULBD using a modified uniportal interlaminar endoscopic system, which had a large-diameter working channel (Fig. 1 lower). Microscopic decompressive surgeries (ULBD) were performed using Caspar lumbar retractor or tubular retractor systems with a microscope. The percutaneous uniportal endoscopic approach utilized only one channel with a single skin incision (Fig. 1 lower). ${ }^{9}$ We made a 10 -mm-long small incision for the surgery.
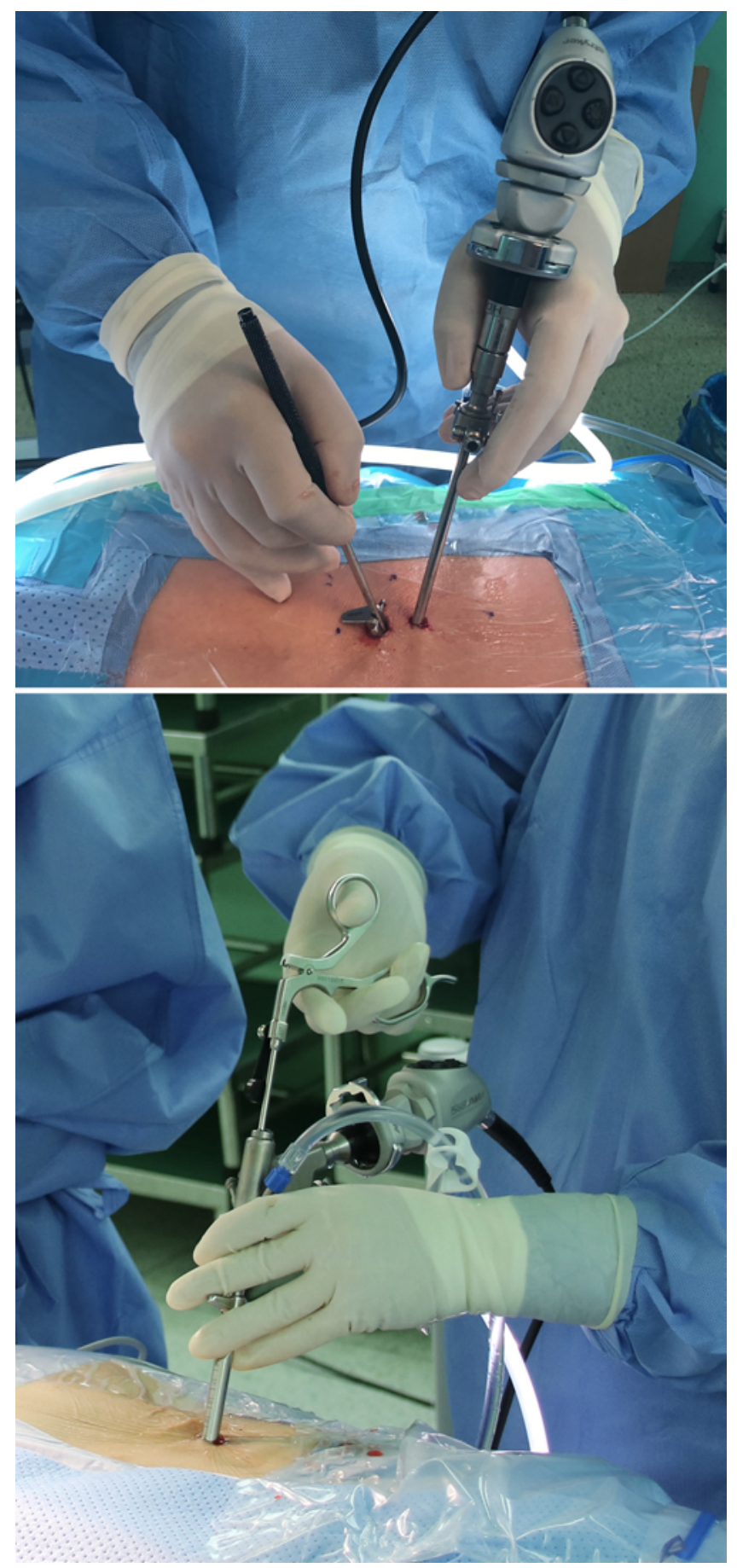

FIG. 1. Intraoperative views of a percutaneous biportal endoscopic approach (upper) and a percutaneous uniportal endoscopic approach (lower).

The same ULBD procedure has been performed using the uniportal interlaminar endoscopic approach. ${ }^{9}$

The percutaneous biportal endoscopic surgeries involved the use of two portals: an endoscopic portal and a working portal (Fig. 1 upper) ${ }^{6,8}$ We made a 5-mm-long skin incision for the endoscopic portal and 7-mm-long skin incision for the working portal. ${ }^{5,6}$ The endoscopic portal was used for endoscopy and its sheath, whereas the 

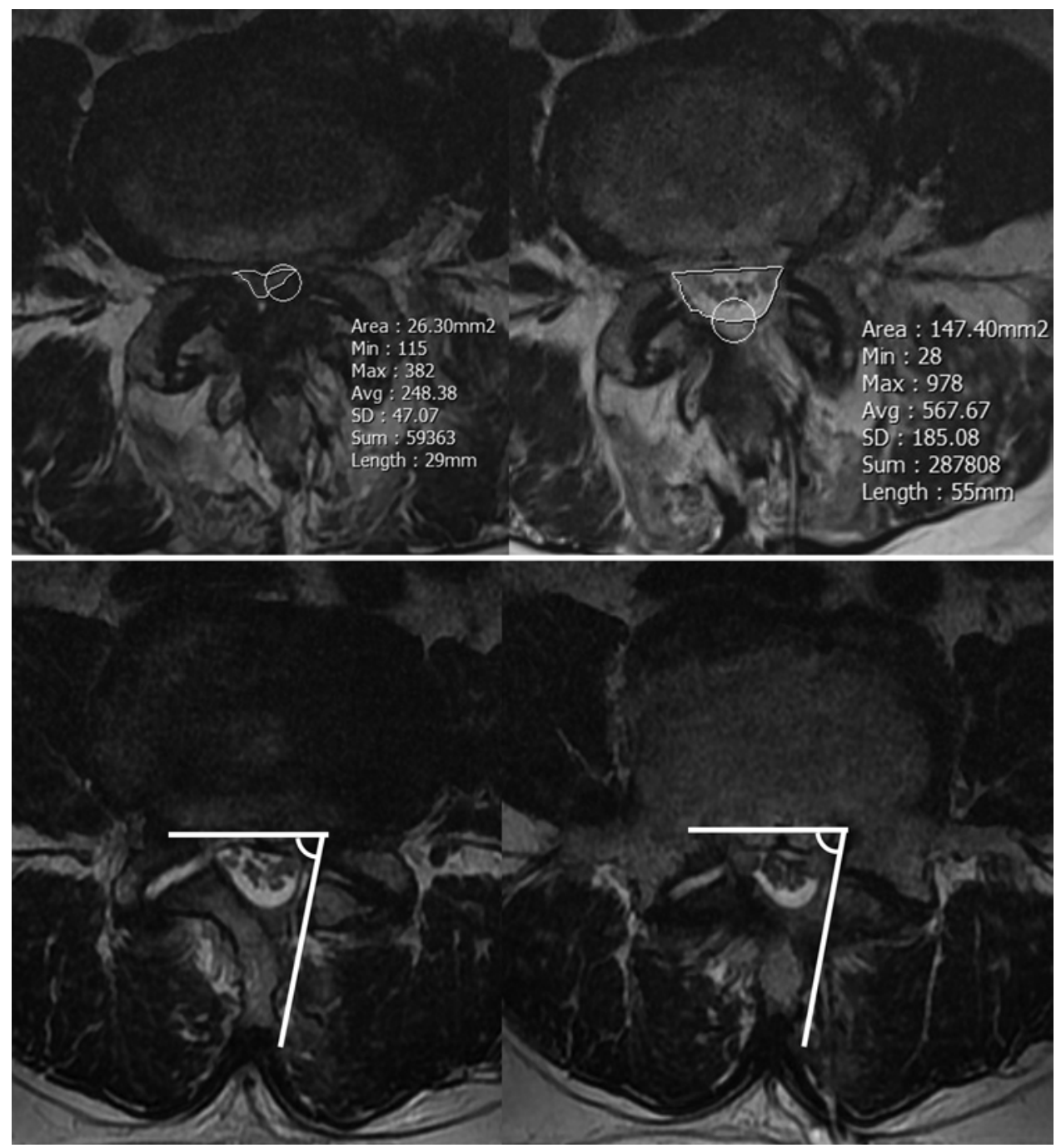

FIG. 2. Measurement of the area of the dural sac and angle of the facetectomy surface. The area was automatically calculated by drawing a line along the outer wall of the dura (upper). Measurement of the angle of the medial surface of the facetectomy (lower).

working portal was used as a channel for spinal operation instruments. ${ }^{8}$ Following partial hemilaminectomy, we always tried to totally remove the ligamentum flavum from the proximal end to the distal end. Contralateral decompression was performed until full exposure of the contralateral descending nerve root (Video 1).

VIDEO 1. Intraoperative video of percutaneous biportal endoscopic decompression for lumbar central stenosis. Contralateral decompression was performed until full exposure of the contralateral descending nerve root. Traversing nerve roots and the central canal were confirmed to be decompressed, and the operation was completed. Copyright Dong Hwa Heo. Published with permission. Click here to view.

\section{Analysis of Radiological and Clinical Parameters}

We classified patients into three groups based on the surgical approach they had undergone: ${ }^{6}$ microsurgery group, biportal endoscopy group, and uniportal endoscopy group.

Magnetic resonance images were obtained the 1st or 2nd day after surgery. The automatic area calculation program of the picture archiving and communication system (Infinitt PACS, Infinitt Healthcare South Korea) was used for measuring the area of the dura (Fig. 2 upper). ${ }^{6}$ If the line was drawn outside of the dura, the area of the dura was automatically calculated by the program. ${ }^{6}$ Axial T2weighted MR images were obtained at 3-mm intervals, and the center of the axial images was in the middle of the intervertebral disc space. The area of the dura was measured on five axial MRI slices preoperatively and postoperatively. Two neurosurgeons blinded to patient information measured the area of the dura twice. ${ }^{6}$ After summing the five areas, the difference between the postoperative area and the preoperative area was calculated to measure dural expansion. ${ }^{6}$ The mean area of dural expansion was compared among the three treatment groups.

We measured the angle of the medial facetectomy area to investigate facet undercutting. We measured the angle of the cutting surface of the facet joint using two axial T2-weighted MRI slices (Fig. 2 lower). An angle $<90^{\circ}$ indicated facet undercutting. We compared the mean angle 
of the cutting surface of the facet joint among the three groups.

The visual analog scale (VAS) score for back pain and leg pain were evaluated preoperatively and 1 day and 1 year or more postoperatively. The Oswestry Disability Index (ODI) was assessed preoperatively and 1 year or more postoperatively. Mean values for the VAS score and ODI were compared among the three groups. Complications related to the surgeries and the mean operation time of each surgical procedure were investigated and compared.

\section{Statistical Analysis}

We used nonparametric statistical analysis. All clinical and radiological parameters were analyzed using the Pearson chi-square test, Wilcoxon signed-rank test, MannWhitney U-test, and Kruskal-Wallis test. A p value $<0.05$ was considered statistically significant. We used R 3.1.2 for Windows (R Foundation for Statistical Computing) for all statistical analyses.

\section{Results}

In the period from March 2016 to December 2017, 168 patients with L4-5 lumbar stenosis were treated with minimally invasive ULBD. Ninety-seven patients among them were followed up for more than 12 months after surgery, including 33 patients who had undergone microscopic decompression, 37 who had undergone percutaneous biportal endoscopy, and 27 who had undergone percutaneous uniportal endoscopy. These 97 patients were included in this study. The mean follow-up period was $12.5 \pm 3.3$ months. There were no significant differences in age or sex among the three groups ( $p>0.05$; Table 1$)$.

\section{Radiological Outcomes}

Dural areas were significantly expanded postoperatively in each of the three groups: from $380.2 \pm 66.9$ to 713.5 $\mathrm{mm}^{2}$ in the microscopy group, from $378.7 \pm 79.2$ to 702.1 $\pm 101 \mathrm{~mm}^{2}$ in the biportal endoscopy group, and from $392.8 \pm 73.6$ to $653.5 \pm 73.4 \mathrm{~mm}^{2}$ in the uniportal endoscopy group (all p < 0.05; Table 1). Preoperatively stenotic dural areas were also significantly expanded after surgery in all three groups (all p $<0.05$; Fig. 3).

Mean differences between postoperative and preoperative cross-sectional areas of the dural sac (dural expansion) were as follows: $333.3 \pm 72.8 \mathrm{~mm}^{2}$ in the microscopy group, $323.4 \pm 69.7 \mathrm{~mm}^{2}$ in the biportal endoscopy group, and $260.7 \pm 45.4 \mathrm{~mm}^{2}$ in the uniportal endoscopy group. Mean dural expansion in the uniportal endoscopy group was significantly lower than that in the microscopy or biportal endoscopy group (both $\mathrm{p}<0.05$; Table 1 ).

The mean angle of facetectomy was $93.6^{\circ} \pm 3.8^{\circ}$ in the microscopy group, $88.9^{\circ} \pm 2.7^{\circ}$ in the biportal endoscopy group, and $92.9^{\circ} \pm 4.3^{\circ}$ in the uniportal endoscopy group. The mean angle of facetectomy in the biportal endoscopy group was significantly lower than that in the microscopy or uniportal endoscopy group (both $\mathrm{p}<0.05$; Table 1 ).

\section{Clinical Outcomes}

The VAS back pain score on postoperative day 1 was
TABLE 1. Comparison of radiological and clinical results among microscopy, biportal endoscopy, and uniportal endoscopy groups

\begin{tabular}{|c|c|c|c|}
\hline Variable & $\begin{array}{l}\text { Microscopy } \\
\text { Group }\end{array}$ & $\begin{array}{c}\text { Biportal } \\
\text { Endoscopy } \\
\text { Group }\end{array}$ & $\begin{array}{c}\text { Uniportal } \\
\text { Endoscopy } \\
\text { Group }\end{array}$ \\
\hline No. of patients & 33 & 37 & 27 \\
\hline Sex: M/F & $12 / 21$ & $15 / 22$ & $11 / 16$ \\
\hline Age in yrs & $63.4 \pm 11.1$ & $66.7 \pm 9.4$ & $67.3 \pm 9.9$ \\
\hline $\begin{array}{l}\text { Dural expansion after op } \\
\text { in } \mathrm{mm}^{2 *}\end{array}$ & $333.3 \pm 72.8$ & $323.4 \pm 69.7$ & $260.7 \pm 45.5$ \\
\hline $\begin{array}{l}\text { Angle of facetecomy } \\
\text { in }{ }^{\circ *}\end{array}$ & $93.6 \pm 3.8$ & $88.9 \pm 2.7$ & $92.9 \pm 4.3$ \\
\hline Mean op time in mins* & $56.4 \pm 4.7$ & $62.4 \pm 5.7$ & $61.6 \pm 3.0$ \\
\hline \multicolumn{4}{|l|}{ VAS back pain score } \\
\hline Preop & $6.64 \pm 1.45$ & $7.02 \pm 1.34$ & $7.04 \pm 1.48$ \\
\hline 1 day after op & $3.39 \pm 1.12$ & $1.78 \pm 0.78$ & $1.74 \pm 0.71$ \\
\hline Final FU & $2.03 \pm 0.92$ & $1.95 \pm 0.81$ & $1.81 \pm 0.68$ \\
\hline \multicolumn{4}{|l|}{ VAS leg pain score } \\
\hline Preop & $7.67 \pm 1.08$ & $8.05 \pm 1.08$ & $7.93 \pm 1.07$ \\
\hline 1 day after op & $2.30 \pm 0.92$ & $1.83 \pm 0.76$ & $2.15 \pm 0.95$ \\
\hline Final FU & $1.94 \pm 0.79$ & $2.16 \pm 0.79$ & $1.89 \pm 0.80$ \\
\hline \multicolumn{4}{|l|}{ ODI } \\
\hline Preop & $56.36 \pm 5.91$ & $58.68 \pm 5.57$ & $56.70 \pm 5.66$ \\
\hline Final FU & $22.58 \pm 4.57$ & $23.14 \pm 2.69$ & $23.54 \pm 2.67$ \\
\hline \multicolumn{4}{|l|}{ Complications } \\
\hline Postop instability & 0 & 0 & 0 \\
\hline Durotomy & 2 & 1 & 1 \\
\hline Transient weakness & 1 & 0 & 1 \\
\hline Postop hematoma & 2 & 1 & 1 \\
\hline
\end{tabular}

significantly higher in the microscopy group than in the uniportal or biportal endoscopy group (both $\mathrm{p}<0.05$; Table 1). However, there were no significant differences in the VAS back pain scores, VAS leg pain scores, or ODI at the final follow-up among the three groups $(\mathrm{p}>0.05)$.

The mean operation time was $56.4 \pm 4.7$ minutes in the microscopy group, $62.4 \pm 5.7$ minutes in the biportal endoscopy group, and $61.6 \pm 3.0$ minutes in the uniportal endoscopy group. The mean operation time in the microscopy group was significantly lower than that in either endoscopy group ( $p<0.05$; Table 1$)$. We experienced perioperative complications such as dural injury, transient weakness on ankle dorsiflexion, and postoperative epidural hematomas. There was no significant difference in the incidence of perioperative complications among the three groups $(\mathrm{p}>0.05)$.

\section{Discussion}

Various kinds of minimally invasive spine surgeries have been attempted for lumbar degenerative disease., ${ }^{2,14}$ Minimally invasive spine surgery can preserve normal structures and lead to fast recovery after operation. ${ }^{2,12,14}$ 

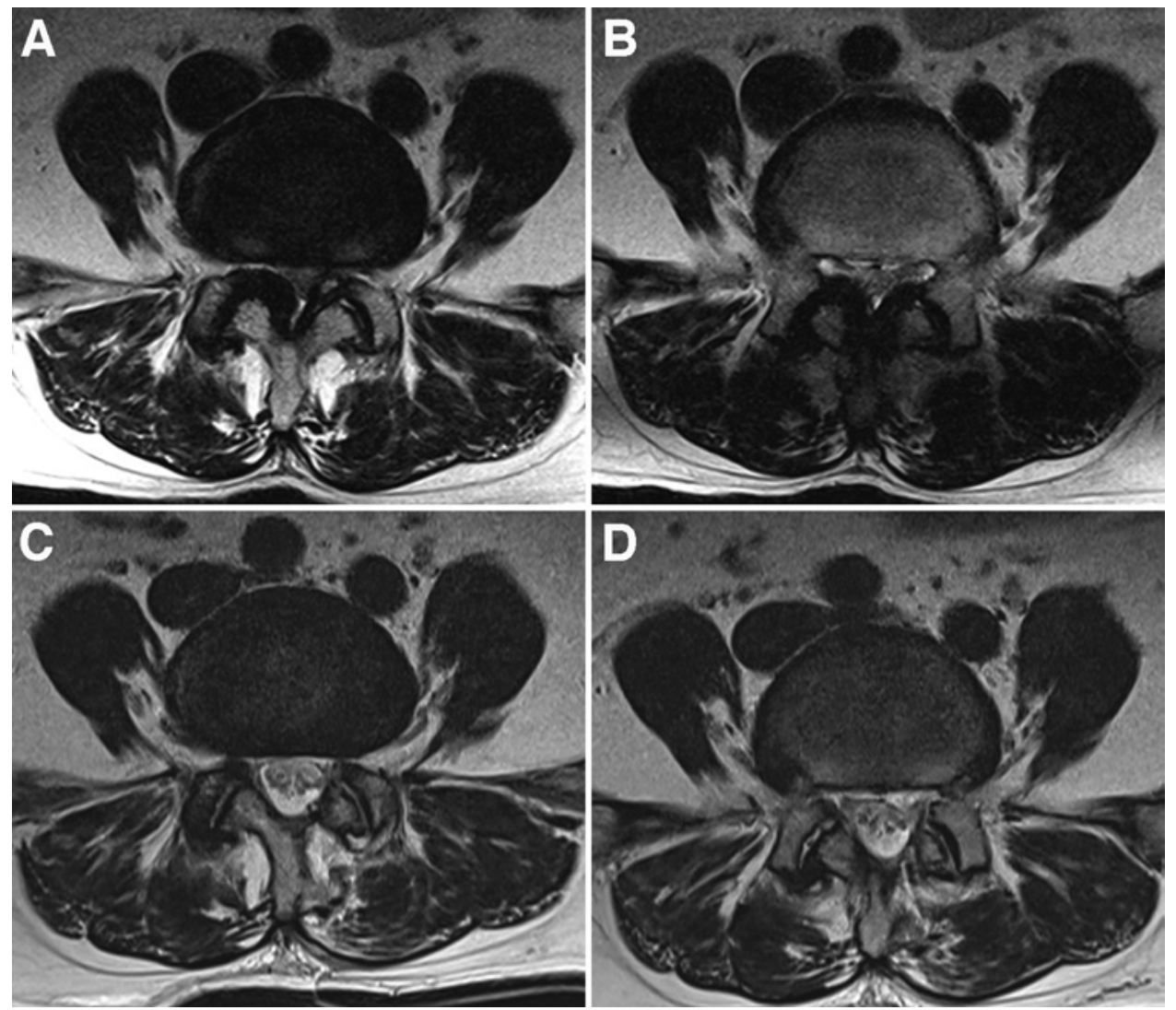

FIG. 3. An 82-year-old female presented with left-sided leg pain with claudication. Preoperative axial T2-weighted MR images showing severe central canal stenosis at $L 4-5$ (A and B). Postoperative MR images showing that the central canal is widely decompressed after percutaneous biportal endoscopic decompression. The left-sided facet joint was undercut and well preserved (C and D).

Among such procedures are percutaneous endoscopic approaches, which usually have been used to treat herniated disc disease. Recently, indications for percutaneous endoscopic spine surgeries have expanded and replaced some microsurgeries. ${ }^{59}$ Lumbar central stenosis has been treated via percutaneous endoscopic approaches. ${ }^{8,12}$ Minimally invasive decompressive surgery like ULBD can be performed using modified interlaminar endoscopic systems. Basically, endoscopic decompressive surgery for lumbar stenosis is the same as the unilateral approach with decompression of bilateral traversing roots as in microscopic ULBD. Minimally invasive ULBD procedures can be performed via the percutaneous uniportal or biportal endoscopic approach. ${ }^{1,8}$ The purpose of endoscopic decompressive surgery for lumbar stenosis is to perform wide decompression of the neural canal and minimize injury to the posterior musculo-ligamentous structures.?

Although favorable clinical outcomes of endoscopic decompressive surgeries have been documented, few studies have reported radiological outcomes or whether the endoscopic approach can achieve enough decompression for lumbar stenosis like other conventional decompressive surgeries. ${ }^{6}$ In the percutaneous biportal endoscopic approach, we have tried to achieve complete decompression. The ligamentum flavum was removed from its proximal to distal end after full exposure was achieved. Ipsilateral laminotomy and medical facetectomy were performed until full exposure of the traversing nerve root. Bilateral medial borders of pedicles were checked to confirm full decompression of the bilateral traversing nerve roots (Video 1). ${ }^{6}$

Dural expansion after endoscopic decompressive procedures was measured using MRI. The degree of dural expansion was significantly increased after the three different decompressive surgeries. There was no significant difference in dural expansion between the microscopic group and the biportal endoscopic group. However, mean dural expansion in the uniportal endoscopy group was significantly lower than that in either the microscopy or biportal endoscopy group. Percutaneous uniportal endoscopic decompression may involve technical difficulty with a steep learning curve and instrument limitations. Reasons for the inferior canal decompression in the uniportal endoscopic surgery compared to that obtained with the other procedures may be related to the restricted use of surgical instruments and the relative difficulty in removing bone since movement of the surgical instruments is possible only for straight forward and back.

Although microscopic decompressive surgery for dural expansion is superior to uniportal or biportal endoscopic surgery, endoscopic decompression may have the advantage of facet preservation (facet undercutting) while mini- 
mizing muscle injury (Fig. 3). Medial facetectomy was necessary for decompression of the ipsilateral traversing nerve root. However, laminotomy with medial facetectomy can induce postoperative segmental instability. ${ }^{4} \mathrm{We}$ measured the angle of the cutting surface in the medial facetectomy for the three groups. Percutaneous biportal endoscopic surgery had better results of facet undercutting than microsurgery (Fig. 1 and Table 1). Biportal endoscopic approaches can use various instruments including conventional ones. Partially curved Kerrison punches, rotation punch, and angled curette can be used to perform undercutting and preserve the ipsilateral facet joint in percutaneous biportal endoscopic surgery. However, based on our experience, facet joint preservation and wide neural decompression seem to somewhat depend on the surgeon's ability rather than the type of surgical approach.

The uniportal or biportal endoscopic approach may have better clinical results in the immediate postoperative periods. This means that surgery-related posterior musculo-ligamentous injury may be lower in the biportal or uniportal endoscopic technique than in the microsurgical procedure, thus allowing for early recovery.

Perioperative complications are also important. There were no statistical differences in the incidence of postoperative complications between the endoscopic and microscopic groups. However, the incidence of surgical complications may be higher in learning-curve periods of the endoscopic spinal approaches. We strongly recommend that endoscopic spine procedures be done with supervision to prevent complications in the learning-curve periods. Although postoperative instability was not found during the follow-up period in our three groups, longer follow-up is needed to evaluate postoperative instability.

\section{Study Limitations}

Our study has several limitations. First, it was a retrospective analysis. Second, it was not a randomized or blinded study. Third, the sample size was small, and the follow-up period was relatively short. In addition, there may be selection bias in determining the surgical procedures. Thus, a randomized controlled study should be performed in the future. Long-term studies in large populations should be conducted to evaluate postoperative muscle atrophy, postoperative segmental instability, restenosis after the first surgery, and long-term clinical outcomes. Moreover, a double-blind study is needed to reduce biases and ensure accurate comparative studies.

\section{Conclusions}

We were able to achieve significant lumbar canal decompression with all three minimally invasive surgical approaches. Microsurgery for lumbar central stenosis may be better than endoscopic approaches for postoperative spinal canal and dural expansion. The percutaneous biportal endoscopic approach may have benefit in terms of avoiding postoperative facet joint violation. Although radiological results were different among the three types of surgeries, clinical outcomes were significantly improved after each type. Percutaneous biportal or uniportal endoscopic approaches may offer the advantage of reduced immediate postoperative pain. A percutaneous uniportal or biportal endoscopic lumbar approach may be effective for the treatment of lumbar central stenosis and an alternative to conventional microsurgical decompression.

\section{References}

1. Ahn Y: Percutaneous endoscopic decompression for lumbar spinal stenosis. Expert Rev Med Devices 11:605-616, 2014

2. Alimi M, Hofstetter CP, Pyo SY, Paulo D, Härtl R: Minimally invasive laminectomy for lumbar spinal stenosis in patients with and without preoperative spondylolisthesis: clinical outcome and reoperation rates. J Neurosurg Spine 22:339-352, 2015

3. Bresnahan LE, Smith JS, Ogden AT, Quinn S, Cybulski GR, Simonian N, et al: Assessment of paraspinal muscle cross-sectional area after lumbar decompression: minimally invasive versus open approaches. Clin Spine Surg 30:E162E168, 2017

4. Guha D, Heary RF, Shamji MF: Iatrogenic spondylolisthesis following laminectomy for degenerative lumbar stenosis: systematic review and current concepts. Neurosurg Focus 39(4):E9, 2015

5. Heo DH, Kim JS, Park CW, Quillo-Olvera J, Park CK: Contralateral sublaminar endoscopic approach for removal of lumbar juxtafacet cysts using percutaneous biportal endoscopic surgery: technical report and preliminary results. World Neurosurg 122:474-479, 2019

6. Heo DH, Quillo-Olvera J, Park CK: Can percutaneous biportal endoscopic surgery achieve enough canal decompression for degenerative lumbar stenosis? Prospective case-control study. World Neurosurg 120:e684-e689, 2018

7. Heo DH, Son SK, Eum JH, Park CK: Fully endoscopic lumbar interbody fusion using a percutaneous unilateral biportal endoscopic technique: technical note and preliminary clinical results. Neurosurg Focus 43(2):E8, 2017

8. Hwa Eum J, Hwa Heo D, Son SK, Park CK: Percutaneous biportal endoscopic decompression for lumbar spinal stenosis: a technical note and preliminary clinical results. J Neurosurg Spine 24:602-607, 2016

9. Kim HS, Paudel B, Jang JS, Oh SH, Lee S, Park JE, et al: Percutaneous full endoscopic bilateral lumbar decompression of spinal stenosis through uniportal-contralateral approach: techniques and preliminary results. World Neurosurg 103:201-209, 2017

10. Kim SK, Kang SS, Hong YH, Park SW, Lee SC: Clinical comparison of unilateral biportal endoscopic technique versus open microdiscectomy for single-level lumbar discectomy: a multicenter, retrospective analysis. J Orthop Surg Res 13:22, 2018

11. Komp M, Hahn P, Merk H, Godolias G, Ruetten S: Bilateral operation of lumbar degenerative central spinal stenosis in full-endoscopic interlaminar technique with unilateral approach: prospective 2-year results of 74 patients. J Spinal Disord Tech 24:281-287, 2011

12. Komp M, Hahn P, Oezdemir S, Giannakopoulos A, Heikenfeld R, Kasch R, et al: Bilateral spinal decompression of lumbar central stenosis with the full-endoscopic interlaminar versus microsurgical laminotomy technique: a prospective, randomized, controlled study. Pain Physician 18:61-70, 2015

13. Poletti CE: Central lumbar stenosis caused by ligamentum flavum: unilateral laminotomy for bilateral ligamentectomy: preliminary report of two cases. Neurosurgery 37:343-347, 1995

14. Storzer B, Schnake KJ: Microscopic bilateral decompression by unilateral approach in spinal stenosis. Eur Spine J 25 (Suppl 2):270-271, 2016

15. Weinstein JN, Tosteson TD, Lurie JD, Tosteson AN, Blood 
E, Hanscom B, et al: Surgical versus nonsurgical therapy for lumbar spinal stenosis. N Engl J Med 358:794-810, 2008

\section{Disclosures}

The authors report no conflict of interest concerning the materials or methods used in this study or the findings specified in this paper.

\section{Author Contributions}

Conception and design: all authors. Acquisition of data: Heo, Lee. Drafting the article: Heo, Park. Critically revising the article: Heo. Reviewed submitted version of manuscript: Heo, Lee. Approved the final version of the manuscript on behalf of all authors: Heo. Study supervision: Park.

\section{Supplemental Information}

Videos

Video 1. https://vimeo.com/322772909.

\section{Correspondence}

Dong Hwa Heo: The Leon Wiltse Memorial Hospital, Gyeonggido, Korea. spinesurgery@naver.com. 